\title{
ENTROPIES AND FACTORIZATIONS OF TOPOLOGICAL MARKOV SHIFTS
}

\author{
BY D. A. LIND 1
}

1. Markov shift entropies. Let $A$ be a nonnegative integral matrix. A well-known construction [7] associates to $A$ a homeomorphism $\sigma_{A}$ of a totally disconnected compact space called a topological Markov shift, or subshift of finite type. Such Markov shifts play a central role in topological dynamics (see [3]), the investigation of Smale's Axiom A diffeomorphisms [6], and coding theory [1]. We announce here a characterization of the possible values for the topological entropy of such Markov shifts, answering a question raised in [2]. Furthermore, these values possess an arithmetic structure which, together with the isomorphism theorem of Adler and Marcus [2], yields an analogue of prime factorization for Markov shifts up to almost topological conjugacy. Details and applications of these results will appear elsewhere.

We shall always assume $A$ to be aperiodic, i.e. some power of $A$ is strictly positive. The topological entropy of $\sigma_{A}$ is $\log \lambda$, where $\lambda$ is the spectral radius of $A$ [5]. Perron-Frobenius theory [4] shows that $\lambda$ must be an algebraic integer $>1$ whose other conjugates have absolute value $<\lambda$. Call an algebraic integer with these properties a Perron number. Our principal result shows these are the only restrictions on Markov shift entropies.

TheOREM 1. If $\lambda$ is a Perron number, then there is a nonnegative aperiodic integral matrix whose spectral radius is $\lambda$.

Sketch of Proof. If $\lambda$ is Perron, let $B$ be the $d \times d$ companion matrix of the minimal polynomial over $\mathbf{Q}$ of $\lambda$. The main difficulty occurs when $B$ has no invariant $d$-sided cones, e.g. when $\operatorname{tr} B<0$. This is overcome by finding invariant surfaces for $B$ curved towards the dominant eigendirection.

The real Jordan form for $B$ decomposes $\mathbf{R}^{d}$ into direct sum of the 1dimensional dominant eigenspace $D=\mathbf{R} w$ for $\lambda$, a collection $\mathcal{E}=\{E\}$ of 1- or 2-dimensional eigenspaces with $\|B x\|=\gamma_{E}\|x\|(x \in E)$ for constants $\gamma_{E}>1$, and another collection $\mathcal{F}=\{F\}$ of eigenspaces with $\|B x\|=\gamma_{F}\|x\|(x \in F)$, $\gamma_{F} \leq 1$. If $G=D, E$, or $F$, let $\pi_{G}$ be the $B$-equivariant projection from $\mathbf{R}^{d}$ to $G$. We will use $\pi_{D}: \mathbf{R}^{d} \rightarrow \mathbf{R} \cong D$ normalized by $\pi_{D} w=1$. Put $\pi_{C}=I-\pi_{D}$.

Fix $\theta>0$, and put

$$
K_{\theta}=\left\{x \in \mathbf{R}^{d}: \pi_{D} x>\theta\left\|\pi_{C} x\right\|\right\}, \quad K_{\theta}(r)=\left\{x \in K_{\theta}: \pi_{D} x \leq r\right\} .
$$

Received by the editors November 16, 1982.

1980 Mathematics Subject Classification. Primary 58F15, 28D20; Secondary 58F11, 58F19.

${ }^{1}$ Supported in part by NSF Grant MCS 8201542. 
For sufficiently large $r$, the semigroup generated by $K_{\theta}(r) \cap \mathbf{Z}^{d}$ contains $K_{2 \theta} \cap$ $\mathbf{Z}^{d}$. Define $\phi: \bigoplus_{\mathcal{E}} E \rightarrow D$ by

$$
\phi\left(\sum_{E} x_{E}\right)=\left(\sum_{E}\left\|x_{E}\right\|^{\log \lambda / \log \gamma_{E}}\right)_{w} .
$$

The graph of $\phi$ is $B$-invariant and bowl-shaped since $\log \lambda / \log \gamma_{E}>1$. Choose $\xi, \eta>0$ so that

$$
K_{\theta}(r) \subset\left\{x \in \mathbf{R}^{d}: \max _{F}\left\|\pi_{F} x\right\| \leq \xi, \pi_{D} \phi\left(\sum_{E} \pi_{E} x\right) \leq \eta \pi_{D} x\right\}=\Omega .
$$

To construct a nonnegative aperiodic integral matrix $A$ with spectral radius $\lambda$, consider $\Gamma=\left\{z \in \Omega \cap \mathbf{Z}^{d}: \pi_{D} z \leq s\right\}=\left\{z_{j}: 1 \leq j \leq n\right\}$, where $s$ is chosen large enough for (ii) below. Write

$$
B z_{i}=\sum_{j=1}^{n} a_{i j} z_{j}
$$

with $a_{i j} \in \mathbf{Z}^{+}$using these rules: (i) if $\pi_{D} z_{i} \leq s / \lambda$, then $B z_{i}=z_{j_{0}} \in \Gamma$ and let $a_{i j}=\delta_{j j_{0}}$; (ii) if $s / \lambda<\pi_{D} z_{i} \leq s$, then $B z_{i}-z_{i} \in K_{2 \theta}$, and therefore is a nonnegative integral combination of elements of $K_{\theta}(r) \cap \mathbf{Z}^{d} \subset \Gamma$ and then the $a_{i j}$ can be chosen with $a_{i i} \geq 1$. This yields $A=\left[a_{i j}\right]$. If $A$ is reducible, replace $A$ by an irreducible component [4] keeping the same notation. Condition (ii) forces $\operatorname{tr} A>0$, so $A$ is aperiodic. Using Perron-Frobenius theory, it can be shown that $A$ has spectral radius $\lambda$.

2. An example. Given a Perron number $\lambda$, this proof provides an algorithm for computing a nonnegative aperiodic integral matrix $A$ with spectral radius $\lambda$. When $\lambda$ has negative trace, the dimension of $A$ must be strictly larger than the degree of $\lambda$. For example, the Perron root $\lambda \cong 3.8916$ of $t^{3}+3 t^{2}-15 t-46$ has conjugates $\lambda_{2} \cong-3.2142, \lambda_{3} \cong-3.6775$ and trace -3 . Using $\eta=1 / 10$ in (1), $\Omega$ was searched for a collection $\Gamma$ of lattice points obeying (2). Such a $\Gamma$ with 10 points was found, giving

$$
A=\left[\begin{array}{rrrrrrrrrr}
0 & 0 & 0 & 0 & 1 & 0 & 0 & 1 & 0 & 0 \\
0 & 0 & 1 & 1 & 0 & 1 & 0 & 0 & 0 & 0 \\
0 & 3 & 0 & 0 & 0 & 1 & 0 & 0 & 0 & 0 \\
3 & 1 & 0 & 0 & 0 & 1 & 0 & 0 & 0 & 0 \\
0 & 0 & 0 & 0 & 0 & 0 & 0 & 0 & 0 & 1 \\
0 & 0 & 0 & 2 & 0 & 0 & 2 & 0 & 0 & 0 \\
0 & 0 & 0 & 0 & 0 & 0 & 0 & 0 & 1 & 0 \\
5 & 0 & 0 & 0 & 0 & 0 & 2 & 0 & 0 & 0 \\
1 & 4 & 0 & 0 & 0 & 0 & 12 & 0 & 0 & 0 \\
0 & 2 & 1 & 0 & 9 & 0 & 0 & 3 & 0 & 0
\end{array}\right]
$$


The characteristic polynomial of $A$ factors over $\mathbf{Q}$ as

$$
(t+1) \times\left(t^{3}+3 t^{2}-15 t-46\right)\left(t^{6}-4 t^{5}-4 t^{4}+27 t^{3}-6 t^{2}-50 t+24\right) .
$$

The roots of the degree 6 irreducible factor are about $0.5134,-1.8277 \pm$ $0.1641 i, 1.9689 \pm 0.6751 i$, and 3.2042 , so the spectral radius of $A$ is indeed $\lambda$.

3. An arithmetic for Perron numbers. Let $\mathbf{P}$ denote the set of Perron numbers. Then $\mathbf{P}$ is closed under addition and multiplication. If $K$ is a finite extension field of $\mathbf{Q}$, it can be shown that $K \cap \mathbf{P}$ is a discrete subset of $[1, \infty)$.

Call $\lambda \in \mathbf{P}$ indecomposable if it cannot be written as $\alpha \beta$ with $\alpha, \beta \in \mathbf{P}$. Thus 2 is indecomposable; for if $2=\alpha \beta$ with $\alpha, \beta \notin \mathbf{Z}$, then a conjugate $\beta_{i}=2 / \alpha_{i}$ of $\beta$ would have $\left|\beta_{i}\right|=2 /\left|\alpha_{i}\right|>2 / \alpha=\beta$, contradicting $\beta \in \mathbf{P}$. A modification due to $\mathrm{M}$. Boyle of this argument proves the following.

Proposition. Let $\lambda=\alpha \beta \in \mathbf{P}$ with $\alpha, \beta \in \mathbf{P}$. Then $\alpha, \beta \in \mathbf{Q}(\lambda)$.

Since $\mathbf{Q}(\lambda) \cap \mathbf{P}$ is discrete, it follows that $\lambda$ can be factored into indecomposables, but in only finitely many ways. The Perron factorization of a rational integer coincides with its usual prime factorization, and is unique by the Proposition. Unfortunately, nonuniqueness can occur, as in $(\alpha+2)^{2}=5 \alpha^{2}$, where $\alpha=(1+\sqrt{5}) / 2$, and each factor is indecomposable.

4. Factorizations of topological Markov shifts. Adler and Marcus [2] introduced the notion of almost topological conjugacy, and proved that two aperiodic Markov shifts with the same entropy are almost topologically conjugate. Together with Theorem 1, this proves the following.

Theorem 2. Let $\sigma$ be an aperiodic topological Markov shift with entropy $\log \lambda$. Then up to almost topological conjugacy, there is a one-to-one correspondence between factorizations $\sigma=\sigma_{1} \times \cdots \times \sigma_{n}$ of $\sigma$ into a direct product of aperiodic Markov shifts and Perron factorizations $\lambda=\lambda_{1} \times \cdots \times \lambda_{n}$ of $\lambda$, where $\lambda_{j} \in \mathbf{P}$. In particular, the number of such factorizations is finite.

Corollary 1. Let $\sigma$ be as in Theorem 2, and assume further that $\lambda$ is indecomposable. Then $\sigma$ is not even almost topologically conjugate to a direct product of nontrivial aperiodic Markov shifts.

Since direct factors of Markov shifts must be sofic, and sofic entropies coincide with Markov shift entropies, we also obtain the following.

CoROllary 2. Let $p$ be a rational prime. The full p-shift cannot be factored into the direct product of homeomorphisms of nontrivial compact spaces.

\section{REFERENCES}

1. R. L. Adler, D. Coppersmith and M. Hassner, Algorithms for sliding block codes, IEEE Trans. Inform. Theory IT-29 (1983), No. 1, 5-22.

2. R. L. Adler and B. Marcus, Topological entropy and equivalence of dynamical systems, Mem. Amer. Math. Soc. No. 219 (1979). 
3. M. Denker, C. Grillenberger and K. Sigmund, Ergodic theory on compact spaces, Lecture Notes in Math., vol. 527, Springer-Verlag, Berlin and New York, 1976.

4. F. R. Gantmacher, The theory of matrices, Vol. II, Chelsea, New York, 1959.

5. W. Parry, Intrinsic Markov chains, Trans. Amer. Math. Soc. 112 (1964), 55-66.

6. S. Smale, Differentiable dynamical systems, Bull. Amer. Math. Soc. 73 (1967), 747-817.

7. R. F. Williams, Classification of subshifts of finite type, Ann. of Math. (2) 98 (1973), 120-153; errata, Ann. of Math. (2) 99 (1974), 380-381.

Department of Mathematics, University of Washington, Seattle, WashingTON 98195 